\section{RF3 ARE MULTIPLE RISK BEHAVIOUR INTERVENTIONS EQUALLY EFFECTIVE FOR ALL ADOLESCENTS? EXAMINING SUBGROUP EFFECTS BY SOCIOECONOMIC STATUS}

L Tinner*, R Campbell, D Caldwell, G MacArthur, M Hickman. Population Health Sciences, University of Bristol, Bristol, UK

\subsection{6/jech-2018-SSMabstracts.92}

Background Multiple risk behaviour (MRB) refers to two or more risk behaviours including smoking, drinking alcohol, poor diet and unsafe sex. Such behaviours are known to cooccur in adolescence.There are increasing public health interventions that address MRB as opposed to isolated behaviours. However, little is known about differential intervention effects by socioeconomic status (SES). There is a need to examine these effects in order to reduce health inequalities. The aim of this study was to examine universal public health interventions targeting adolescent multiple risk behaviour forsubgroup effects by SES.

Methods Two Cochrane systematic reviews that focused on adolescent MRB were screened to identify universal interventions that reported SES. Study authors were contacted, and outcome data requested stratified by SES and intervention status. Risk behaviour outcomes: alcohol use, smoking, substance use, unsafe sex, overweight/obesity, sedentarism, peer violence and dating violence were examined in random effects metaanalyses and subgroup analyses performed to explore differences between high SES and low SES adolescents.

Results Of 50 studies reporting universal interventions, 15 also reported having measured SES. Of these 15 studies, four study authors provided additional data for subgroup analyses. For alcohol use, the point estimates suggest that SES does not explain the effect of the intervention, as the direction of effect is the same for both high SES (RR 1.28, 95\% CI 0.97, 1.69) and low SES (RR 1.14, 95\% CI 0.97, 1.34). The point estimates for smoking behaviour are indicative of a differential intervention effect in favour of the low SES group (RR 0.83, $95 \%$ CI 0.66, 1.03) versus the high SES group (RR 1.10, 95\% CI 0.78, 1.56). SES was not an explanatory factor for the intervention effect on substance use as the direction of effect in the high SES group (RR 1.13, 95\% CI 0.83, 1.53) and the low SES group (RR 1.26, 95\% CI 0.83, 1.92) was the same. Tests for subgroup differences showed no evidence of difference for all behaviour outcomes.

Conclusion The majority of studies identified did not report having measured SES. Findings from the four studies included in the subgroup analysis indicate the potential for interventions to differentially effect different SES groups. There is a need for routine reporting of demographic information within studies so that stronger evidence of effect by SES can be demonstrated and that interventions can be evaluated for their impact on health inequalities.

\section{RF4 'COMMON VERSUS SPECIFIC LIABILITY FOR SUBSTANCE ABUSE IN EARLY ADULTHOOD: A GENETICALLY INFORMED APPROACH'}

${ }^{1} \mathrm{E} \mathrm{lob}^{*},{ }^{2} \mathrm{~T}$ Schoeler, ${ }^{2} \mathrm{~J}$ Pingault. ${ }^{1}$ Department of Epidemiology and Public Health, University College London, London, UK; ${ }^{2}$ Department of Clinical, Educational and Health Psychology, University College London, London, UK

10.1136/jech-2018-SSMabstracts.93
Background Young people experiencing psychological or behavioural problems are more likely to engage in poly-substance use. The common liability theory posits a common liability to all substance addictions. Such shared risk factors may therefore explain the observed patterns of comorbid substance use, where a common genetic susceptibility could underlie this co-occurrence. However, to date most research has failed to measure the aforementioned common liability, and the first genome-wide association studies (GWAS) have identified genetic variants specific to each substance rather than common ones. Therefore, the aim of this project is twofold: firstly, to construct a new longitudinal measurement model of common liability to substance use; secondly, to investigate specific versus common genetic influences on substance use applying polygenic scoring.

Methods The sample includes 6399 young adults from the Avon Longitudinal Study of Parents and Children. Selfreported data on substance use (i.e. tobacco, alcohol, marijuana, and other illicit drugs) was collected at age 17.5, 20, and 22 with validated questionnaires. A Trait-State-Occasion model applied within Structural Equation Modelling (SEM) was used to identify a common trait factor for substance use across the three time points, as well as occasion- and drugspecific latent factors. Polygenic scores were calculated using GWAS results on substance use and related psychopathological, behavioural, and cognitive phenotypes. All analyses were conducted using $R$ and PRSice software.

Results In Study 1, the Trait-State-Occasion model was tested using Robust Maximum Likelihood Estimation, which accounted for the non-normal distribution of the substance abuse measures. The model fitted the data well, $\chi^{2}$ (33) $=100.572, \quad \mathrm{p}<0.001, \quad C F I=0.987, \quad$ RMSEA $=0.018$, $S R M R=0.027$. The variance of the trait factor was 0.155 [95\% CI 0.124; 0.185]. On average, the model R-Squared was $70 \%$, with residuals being $30 \%$. The total variance explained by the model was further decomposed into trait, occasion, and drug-specific variance, which was equal to $16 \%$, $11 \%$, and $44 \%$ respectively. Study 2 (ongoing) will test a full SEM model by regressing the polygenic scores on both the common trait and drug-specific factors.

Conclusion The results of Study 1 provided evidence for a common liability to substance use, which is partly shared across different drugs and stable over time. This therefore suggests that both common and drug-specific susceptibility should be considered where examining genetic and environmental risk factors for substance use. Study 2 will reveal the influence of genetic variants associated with various psychopathology related-traits on both common and drug-specific dimensions of substance use.

\section{RF5 DIFFERENT CLUSTERS OF RISK AND ADVERSE SEXUAL HEALTH OUTCOMES IN THE BRITISH POPULATION}

MW Waltenberger*, AP Parkes, KM Mitchell. Social Relationships and Health Improvement, Social and Public Health Sciences Unit, Glasgow, UK

\subsection{6/jech-2018-SSMabstracts.94}

Background Although many people enjoy good sexual health, others experience adverse outcomes including: sexually transmitted infections, sexual function problems and sexual coercion. Little is known about whether different combinations of adverse outcomes are present in different groups of people, 
and whether these have implications for mental and sexual wellbeing.

We aimed to identify different clusters of adverse outcomes, to investigate associated socio-demographic and lifestyle factors, and to compare risk of depression and low sexual wellbeing (dissatisfaction and distress with sex life) between groups.

Methods We used data from the British National Surveys of Sexual Attitudes and Lifestyles (NATSAL 3, 2010-2012; men $\mathrm{n}=5113$; women $\mathrm{n}=7019$; ages 16-74). Latent Class Analysis (Mplus, version 8) used 16 variables relating to sexually transmitted infections (and associated sexual risk behaviours and attitudes), sexual coercion and sexual function problems, with men and women analysed separately. Multinomial logistic regression (Stata/SE14.2) assessed factors associated with class membership.

Results We found four groups for men, and six for women. Male groups were: low risk/problems (81\%), sexual function problems (9\%), worried risk-takers (5\%) and unworried risktakers (5\%). Female groups were: low risk/problems (60\%), sexual function problems (7\%), worried risk-takers (3\%), unworried risk-takers (8\%), sex-avoiding (20\%) and high vulnerability (2\%). Unworried risk-takers did not perceive themselves as being at risk, whereas worried risk-takers did. Unworried were more likely than worried risk-takers to be older (men: OR 2.2; 95\% CI 1.1 to 4.2), or smokers (women: OR 1.7, 95\% CI 1.1 to 2.6). The high vulnerability group (found in women only) reported sexual risk, sexual function problems and coercion, and was characterised by drug and alcohol use (compared to low risk/problems group, OR 3.5, CI 1.5-8.3). Compared to low risk/problems groups, other groups were more likely to be depressed, distressed and dissatisfied with their sex life, with odds ratios (all $\mathrm{p}<0.05$ ) for different groups ranged as follows: depression: men 2.13.5, women 2.9-8.4; distressed: men 1.5-4.9, women 3.213.9; dissatisfied: men 2.6 (only sexual function problems group $\mathrm{p}<0.05$ ), women 2.1-11.9. The highest odds occurred among women in the high vulnerability and sexual function problems groups.

Conclusion Identification of different sexual risk/problem groups, all at risk of depression and low sexual well-being, is helpful for planning sexual health policies and services. Of particular interest are two distinct groups of risk-takers (worried and unworried), and a group of women (but not men) who are vulnerable to a range of adverse sexual health outcomes and warrant particular public health attention.

\section{RF6 INEQUALITIES IN NON-INITIATION OF HPV VACCINE: CROSS-SECTIONAL FINDINGS FROM A UK COHORT}

${ }^{1} \mathrm{~N}$ Firman*, ${ }^{2} \mathrm{H}$ Bedford, ${ }^{1} \mathrm{C}$ Dezateux. ${ }^{1}$ Centre for Primary Care and Public Health, Barts and the London School of Medicine and Dentistry, Queen Mary University, London, UK; ${ }^{2}$ Population, Policy and Practice Programme, Great Ormond Street Institute of Child Health, University College London, London, UK

\subsection{6/jech-2018-SSMabstracts.95}

Background HPV vaccination (HPVv) was introduced in the UK in 2008; currently $87 \%-93 \%$ of teenage girls receive at least one dose in school. Uptake is lower in more deprived areas, and, small regional studies report, among Black and minority ethnic groups. Associations with parental and household factors, and school attendance are less clear. Using data from a UK prospective cohort we tested the hypothesis that $\mathrm{HPV}$ initiation is lower among those with parents from Black and minority ethnic groups, living in low income households, and not attending school.

Methods We estimated the percentage of 569014 year-old girls participating in the Millennium Cohort Study whose parent reported $\mathrm{HPVv}$ initiation. We used logistic regression to calculate crude and adjusted odds ratios (OR) of HPVv initiation and examined associations with parental ethnic group (baseline White), school type (non-fee-paying (baseline)/fee-paying/no school), history of school exclusion (baseline no exclusions), and household income (OECD quintile (baseline highest quintile)). Analyses were weighted for survey design (Stata: Release 15; StataCorp LP).

Results 5265 girls (weighted percentage: 92.3w\%; 95\% CI $91.3,93.2)$ received at least one dose of HPVv; $399(7.2 \mathrm{w} \%$; $6.4,8.1)$ no doses; $26(0.5 \mathrm{w} \% ; 0.3,0.9)$ not known. Parents from Bangladeshi (86.1w\%; 80.3, 90.4), Black African (84.9w $\% ; 75.7,91.0)$ and 'other' ethnic groups $(81.0 \mathrm{w} \% ; 70.4$, 88.4) were less likely to report $\mathrm{HPVv}$ initiation compared to those of White ethnicity $(93.6 \mathrm{w} \% ; 92.5,94.5)$. HPVv initiation was lower in girls not attending school $(61.1 \mathrm{w} \% ; 32.5$, 83.7) and those previously excluded from school $(85.2 \mathrm{w} \%$; 78.9, 89.9). After adjusting for age, ethnicity, school type, exclusions and household income, girls with parents from Bangladeshi (OR: 0.57; 0.35, 0.93), Black African (OR: 0.43; $0.23,0.80$ ) or 'other' ethnic groups (OR: 0.30; 0.16, 0.58), those not attending school (OR: $0.11 ; 0.04,0.34$ ), with a history of school exclusion (OR: $0.48 ; 0.30,0.78$ ), or living in low income households (lowest two OECD quintiles OR: $0.46 ; 0.31,0.67$ and OR: $0.51 ; 0.34,0.76)$, were less likely to initiate HPVv.

Conclusion In the UK, there are marked inequalities in $\mathrm{HPVV}$ initiation, with lower uptake among children from poorer households, with parents from Bangladeshi, Black African or other ethnic groups, and those previously excluded or not currently in school. This is the first report of HPVv initiation using a nationally representative cohort. Further work is needed to evaluate interventions for HPVv catch-up in the groups we have identified, who may also be at greater risk of missing cervical screening. Understanding reasons for non-initiation and developing interventions to engage parents from these groups is central to reducing inequalities in HPVv uptake.

\section{RF7 PREVALENCE OF CHRONIC OBSTRUCTIVE PULMONARY DISEASE (COPD) IN GREATER GLASGOW AND CLYDE: AN ECOLOGICAL STUDY BY AGE, SEX, SOCIOECONOMIC AND SMOKING STATUS}

${ }^{1} \mathrm{KA}$ Levin*, ${ }^{2} \mathrm{M}$ Milligan, ${ }^{2} \mathrm{D}$ Anderson. ${ }^{1}$ Public Health Directorate, NHS GGC, Glasgow, UK; ${ }^{2}$ Community Respiratory Team, Glasgow, UK

\subsection{6/jech-2018-SSMabstracts.96}

Background Previously it was estimated that Greater Glasgow and Clyde (GGC) had a COPD prevalence of 2.4\%. COPD has traditionally been associated with males and those from deprived areas. Socioeconomic inequalities in COPD have been largely linked to socioeconomic inequalities in smoking, the most important risk factor for COPD in high income countries. This study aims to calculate the prevalence of COPD in GGC by age, sex, and SES adjusting for smoking status. 